\title{
PREVENÇÃO DE ESCARA DE DECUBITO: AVALIAÇÃO DE PROGRAMA DE TREINAMENTO
}

\author{
Maria Goretti Angarten * \\ Carlos Alberto Monteiro Costa ** \\ Julieta Brasil Bloch **
}

ReBEn/04

ANGARTEN, M.G. e Colaboradores - Prevenção de Escara de Decúbito: Avaliação de Programa de Treinamento. Rev. Bras. Enf.: Rs, 36: 25-28 , 1983.

\section{INTRODUÇÃO}

Os temas relacionados à prevenção de escara de decúbito são causa de grande controvérsia, fato que pode ser confirmado pela falta de uniformidade de cuidados de prevenção de escara de decúbito na maioria dos hospitais, ou mesmo em uma unidade de internação, e pelo número de publicações sugerindo novos métodos e procedimentos de prevenção de escara de decúbito ${ }^{4}$; porém a incidência de escara de decúbito, por muitas vezes encarada como medida de qualidade do Serviço de Enfermagem $^{3,13}$,é um índice variável dependendo das características do grupo de pacientes estudado.

WILLIAMS 16 encontrou a incidência de escara de $2,69 \%$ em 26 pacientes não ambulantes com afecção grave. Pacientes geriátricos foram estudados por NORTON1 2 e $20 \%$ deles desenvolveram escara durante a internação. LOWTHIAN11, em estudo de prevalência de escara de decúbito, encontrou $7 \%$ de 186 pacientes de um hospital ortopédico com escaras de decúbito. LOWTHIAN9, também estudou pacientes de unidades geriátricas e mostrou que $42,0 \%$ dos pacientes internados que permaneciam sentados desenvolveram escara de decúbito nas nádegas. RUBIN e $\operatorname{col}^{14}$ realizaram um trabalho retrospectivo em um hospital geral, encontrando dentre 18000 pacientes admitidos a incidência de $1,46 \%$ IRVINE 7 estudou pacientes incontinentes e em $28,0 \%$ deles escara estava presente. $O$ estudo de SPENCE e col 15 revela que as escaras de decúbito ocorrem em aproximadamente $80,0 \%$ dos pacientes com lesão de coluna espinal e em $10 \%$ a $15 \%$ dos outros pacientes restritos ao leito. GERSON6 relata a incidência de escara de $2,69 \%$ em pacientes de hospital de afecções agudas. ANGARTEN e $\operatorname{col}^{1}$ estudaram pacientes submetidas a tratamento radioterápico na modalidade de radiomoldagem por neoplasia uterina que após três dias de tratamento a incidência de escara foi de $52,4 \%$.

No Hospital Osvaldo Cruz (HOC), a incidência de escara de decúbito medida em um dia do mês de abril de 1982 foi de $14,6 \%{ }^{2}$, segundo o critério de classificação de escara de RUBIN e col ${ }^{14}$. Este índice é várias vezes maior que os relatados por RUBIN e col14 e GERSON6. Este achado tornounecessária medida que intensificasse os esforços para prevenção de escara de decúbito. A solução proposta em reunião do Serviço de Enfermagem foi a de que se realizasse programa de treinamento com a colaboração dos fisioterapeutas sobre a técnica de mudança de decúbito e massagem de conforto para todo o pessoal de enfermagem do HOC.

O objetivo do presente trabalho é avaliar a influência deste programa de treinamento sobre a incidência de escara de decúbito no HOC.

* Enfermeira do Hospital Osvaldo Cruz, São Paulo - SP

* Fisioterapeuta do Hospital Osvaldo Cruz, São Paulo - SP 
ANGARTEN, M.G. e Colaboradores - Prevenção de Escara de Decúbito: Avaliação de Programa de Treinamento. Rev. Bras. Enf.: RS, 36: 25-28, 1983.

\section{DESENVOLVIMENTO}

Programa de treinamento -

O objetivo deste programa foi padronizar minuciosamente a técnica de mudança de decúbito e massagem de conforto e estabelecer que esses cuidados seriam dispensados aos pacientes a cada duas horas. Todos os enfermeiros, técnicos, auxiliares e atendentes de enfermagem participaram da aula teórico-prática ministrada pelos autores.

Avaliação do programa de treinamento -

Seguiu-se o mesmo procedimento usado para medir a incidência de escara de decúbito que motivou o programa de treinamento em uma quinta-feira quatro semanas após o treinamento. Três enfermeiras examinaram a pele da região de apoio de todos os pacientes, ambulantes e acamados, internados no HOC que estavam na unidade de internação na hora da avaliação. $\mathrm{O}$ HOC tem capacidade de 170 leitos, está localizado na área central do Município de São Paulo (SP) e cerca de 80,0\% dos pacientes são da clínica cinúrgica, ortopédica e neurológica.

No exame dos pacientes procurou-se verificar a presença ou não de escara, que foi considerada presente quando se observou as características de uma das três fases de escara descritas por RUBIN e $\operatorname{col}^{14}$ (quadro 1), e se estavam ou não com programa de mudança de decúbito e massagem de conforto.

Quadro 1 - Classificação das escaras de decúbito segundo RUBIN e col ${ }^{14}$.

\begin{tabular}{l}
1 a fase - escara incipiente, área avermelhada com pele íntegra \\
2 a fase - escara com formação de bolha ou úlcera \\
3 ạ fase - escara com necrose, odor fétido ou ambos \\
\hline
\end{tabular}

\section{RESULTADOS}

Dos 118 pacientes que estavam internados no dia da avaliação, oito não foram examinados. Três deles estavam em tratamento com radiomoldagem e cinco pacientes estavam no Centro Cirúrgico.

Vinte e dois tinham escara, significando incidência de escara de $20 \%$. Destes, 16 não estavam sendo submetidos a mudança de decúbito e massagem de conforto. Dois terços dos pacientes que estavam sendo submetidos a massagem de conforto e mudança de decúbito, apresentaram escara de decúbito (tabela 1).

Tabela 1 - Distribuição dos pacientes segundo o cuidado recebido e as condições da pele.

\begin{tabular}{|l|c|c|c|c|c|c|}
\hline \multirow{3}{*}{$\begin{array}{c}\text { CONDIÇÃO DA } \\
\text { PELE }\end{array}$} & \multicolumn{5}{|c|}{ MUDANÇA DE DECÚBITO E MASSAGEM DE } \\
CONFORTO
\end{tabular}

\section{DISCUSSÃO}

A verificação de presença de escara neste trabalho foi baseada na classificação de escara usada por Rubin e col. Esta classificação diferencia-se de outras, pois inclui o eritema persistente como a 1 a das fases na evolução da escara. LOWTHIAN 11 ao discutir a inclusão do eritema persistente na classificação de escara concorda em considerá-lo como escara inicial, porém acha que a sua diferenciação de outras lesões da pele é difícil, justificando assim não incluir este tipo de escara nos seus trabathos. Esta dificuldade em se fazer diagnóstico de escara de 1 a fase (eritema persistente) foi superada pelos autores treinando os observadores, além do fato de que já tinham experiência anterior em estu- 
ANGARTEN, M.G. e Colaboradores - Prevenção de Escara de Decúbito: Avaliação de Programa de Treinamento. Rev. Bras. Enf.: Rs, 36: 25-28 , 1983.

dos semelhantes. Esta opção também buscou encontrar incidência de escara o mais próximo do real, por entenderem os autores que esta seria o modo de interpretação mais adequado quando se avalia procedimento de prevenção.

A incidência de escara observada neste estudo (20\%) é comparável com a relatada anteriormente $(14,6 \%)^{2}$, ambas muito altas quando cotejadas com as incidências observadas por GERSON6 $(2,69 \%)$ e RUB IN 14 (1,46\%) em populações semelhantes à presente. Em grupos de pacientes com patologia ortopédica, onde é necessário maior esforço do pessoal de enfermagem direcionada à prevenção de escara, a incidência observada é da ordem de $7 \%$ (LOWTHIAN ${ }^{11}$ ), ainda ạbaixo ao deste estudo. A incidência aqui relatada encontra paralelo nos estudos de pacientes geriátricos 4,12 .

A tabela 1 mostra que somente 9 pacientes eram submetidos a mudança de decúbito e massagem de conforto. Também pode ser observada que a maior parte $(16 / 22)$ dos pacientes que tiveram escara não eram submetidos a esses cuidados. A avaliação inicialmente proposta - a de quantificar a influência do programa de treinamento sobre incidência de escara - é difícil de ser realizada, pois grande parte dos pacientes que necessitavam desses cuidados de prevenção de escara não o tive$\mathrm{ram}^{2}$. Se se considerar a proporção de pacientes sem escara ${ }^{3}$ entre os que foram submetidos a mudança de decúbito e massagem de conforto ${ }^{9}$ como índice de eficiência da aplicação desses cuidados, talvez incorra-se em erro. Esses pacientes podem ter sido escolhidos para receberem cuidados de prevenção de escara pelas enfermeiras das unidades de internação por serem pacientes com risco maior e muito evidente de desenvolver escara de decúbito, apesar da literatura ser unânime em sugerir que tal incidência $(66,6 \%)$ não seria encontrável entre pacientes submetidos a mudança de decúbito e massagem de conforto $5,8,10,13$.

\section{CONCLUSÃO}

A incidência de escara de decúbito observada no Hospital Osvaldo Cruz um mês após programa de treinamento sobre técnica de prevenção de escara de decúbito é muito alta (20\%).

$72,7 \%$ dos pacientes que tinham escara de decúbito não estavam submetidos aos cuidados de prevenção de escara, indicando que a avaliação clínica do risco de o paciente desenvolver escara realizado pela enfermeira da unidade de internação é inadequada.

A alta incidência de escara de decúbito $(66,6 \%)$ observada entre os pacientes submetidos aos cuidados de prevenção de escara talvez seja explicada pelo insuficiente aproveitamento do programa de treinamento pelo pessoal de enfermagem.

\section{RECOMENDAÇÄO}

Os autores sugerem a definição de critério de avaliação de risco de desenvolvimento de escara para melhor selecionar os pacientes que devem ser submetidos a cuidados de prevenção de escara de decúbito. Sugerem também a padronização da mudança de decúbito em todo o hospital, associando o decúbito a o horário para facilitar a supervisão desses cuidados.

\section{RESUMO}

A incidência de escara de decúbito no Hospital Osvaldo Cruz (SP) era 14,6\%. Este índice justificou o treinamento de pessoal de enfermagem em prevenção de escara. O treinamento compreendeu aula teórico-prática abordando minuciosamente técnica de Mudança de decúbito (MDec) e Massagem de conforto (MCon). Um mês após, a incidência era $20 \%$. Desses 22 pacientes com escara, apenas 6 eram submetidos a MDec e MCon. Estas observações sugerem que o domínio da técnica de MDec e MCon não é suficiente para prevenção de escara. Sugerem os autores a adoção de avaliação padronizada pelo enfermeiro do risco de o paciente desenvolver escara, e associação no hospital do decúbito ao horário visando facilitar a supervisão desses cuidados pelo enfermeiro.

\section{ABSTRACT}

The incidence of pressure sore at Hospital Osvaldo Cruz (São Paulo, Brazil) was $14,6 \%$. This fact justified a pressure sore prevention program. The nursing staf $f$ training program consisted in theory-and-practice classes about turning patients and massage of the lay face of the body. A month after, the incidence was $20 \%$. Only six of these patients had been turned. The authors conclude that the knowledge of how to turn patients isn't enouth for preventing pressure sores and the nurses have 
ANGARTEN, M.G. e Colaboradores - Prevenção de Escara de Decúbito: Avaliação de Programa de Treinamento. Rev. Bras. Enf.: RS, 36: 25-28, 1983.

failed to select the patients that need turning schedule. The outhors suggest that the staff adopt a standard risk estimation for pressure sore risk development and a standardization of hour of the daypatient position schedule throughout the hospital, thus facilitating supervision.

\section{REFERÊNCIAS BIBLIOGRẢFICAS}

1. ANGARTEN, M. G. \& SANTOS, M. L. F. Detecção de alterações em exame físico da pele da região de apoio de pacientes submetidos prolongadamente a um mesmo decúbito. Rev. Bras. Enf., D. F., 33(4). 443-52, 1980.

2. ANGARTEN, M. G. et alii. Incidência de escara de decúbito em hospital geral. (não publicado).

3. BLISS, M. et alii. The use of ripple beds. Nurs. Times, London, 75(7): 280-3, Feb. 15, 1979.

4. EXTON-SMITH, A. N. et alii. Use of the air wave system to prevent pressure sores in hospital. Lancet, London, 1(8284): 1288-90, Jun. 1982.

5. FEUSTEL, Delycia E. Pressure sore prevention. Nurs, 12(4): 78-83, Apr. 1982.

6. GERSON, L. W. The incidence of pressure sores in active treatment hospitals. Int. J. Nurs. Stud, Oxford, 12(4). 201, Oct. 1975.

7. IRVINE, R. E. Norethandrolone and preventing of pressure sores. Lancet, London, 2: 1333, 1961.

8. KAVCHAK - KEYES, M. A. Four proven steps for preventing decubitus ulcers. Nurs, Oxford, 7(9): 58-61, Sep. 1977.

9. LOWTHIAN, (1976), apud: LOWTHIAN, P. T. A review of pressure sore prophylaxis. Nurs, Mirror, Sussex, 144(14), suppl: vii-xv, Mar. 1977.

10. . A review of pressure sore prophylaxis. Nurs. Mirror, Sussex, 144(14), suppl. vii-xv, Mar. 1977.

11. . Pressure sore prevalence. Nurs. Times, London, 75(11). 358-60, Mar. 1, 1979.

12. NORTON, D. et alii. An investigation of geriatric-nursing problems in hospitals. London, National Corporation for the Care of Old People, 1962.p. 325.

13. PRESSURE, sores. A national problem. Nurs. Times, London, 72(8). 290, Feb. 26.1976.

14. RUBIN, C. F. et alii. Auditing the decubitus ulcer problem. Am. J. Nurs, New York, 74(10). 1820-1, Oct. 1974.

15. SPENCE, W. R. et alii. Gel support for prevention of decubitus ulcers. Arch. Phys. Med. Rehabil, 48:283-8, Jun. 1967.

16.WIILIAMS, A.A study of factors contributing to skin breakdow. Nurs. Res, New York, 21(3): 23843, May/Jun. 1972. 